\title{
Six-month exercise training program to treat post-thrombotic syndrome: a randomized controlled two-centre trial
}

\author{
Susan R. Kahn MD MSc, Ian Shrier MD PhD, Stan Shapiro PhD, Adrielle H. Houweling MSc, \\ Andrew M. Hirsch MD, Robert D. Reid PhD MBA, Clive Kearon MB PhD, Khalil Rabhi PhD, \\ Marc A. Rodger MD MSc, Michael J. Kovacs MD, David R. Anderson MD, Philip S. Wells MD MSc
}

\begin{abstract}
Background: Exercise training may have the potential to improve post-thrombotic syndrome, a frequent, chronic complication of deep venous thrombosis. We conducted a randomized controlled two-centre pilot trial to assess the feasibility of a multicentre-based evaluation of a six-month exercise training program to treat post-thrombotic syndrome and to obtain preliminary data on the effectiveness of such a program.
\end{abstract}

Methods: Patients were randomized to receive exercise training (a six-month trainersupervised program) or control treatment (an education session with monthly phone followups). Levels of eligibility, consent, adherence and retention were used as indicators of study feasibility. Primary outcomes were change from baseline to six months in venous diseasespecific quality of life (as measured using the Venous Insufficiency Epidemiological and Economic Study Quality of Life [VEINES-QOL] questionnaire) and severity of post-thrombotic syndrome (as measured by scores on the Villalta scale) in the exercise training group versus the control group, assessed by $t$ tests. Secondary outcomes were change in generic quality of life (as measured using the ShortForm Health Survey-36 [SF-36] questionnaire), category of severity of post-thrombotic syndrome, leg strength, leg flexibility and time on treadmill.

Results: Of 95 patients with post-thrombotic syndrome, 69 were eligible, 43 consented and were randomized, and 39 completed the study. Exercise training was associated with improvement in VEINES-QOL scores (exercise training mean change 6.0, standard deviation [SD] $5.1 \mathrm{v}$. control mean change 1.4, SD 7.2; difference $4.6,95 \% \mathrm{Cl} 0.54$ to $8.7 ; p=0.027$ ) and improvement in scores on the Villalta scale (exercise training mean change -3.6 , SD 3.7 v. control mean change -1.6, SD 4.3; difference $-2.0,95 \%$ $\mathrm{Cl}-4.6$ to $0.6 ; p=0.14$ ). Most secondary outcomes also showed greater improvement in the exercise training group.

Interpretation: Exercise training may improve post-thrombotic syndrome. It would be feasible to definitively evaluate exercise training as a treatment for post-thrombotic syndrome in a large multicentre trial. (Trial registered at www .controlled-trials.com, no. ISRCTN56430072.)

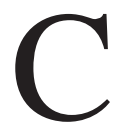
hronic post-thrombotic syndrome develops in up to one-half of patients with deep venous thrombosis and is associated with varying combinations of leg pain, heaviness, swelling, edema, hyperpigmentation and varicose collateral veins. In severe instances, lipodermatosclerosis and venous ulcers occur. ${ }^{1}$ Patients with post-thrombotic syndrome have substantially impaired quality of life. ., $^{2,3}$ Given that effective treatments are lacking, new approaches to managing post-thrombotic syndrome are needed. ${ }^{4}$

Exercise training is an effective treatment for arterial claudication ${ }^{5,6}$ and may also improve post-thrombotic syndrome. ${ }^{7}$ Potential mechanisms include improved endurance resulting from increased aerobic capacity, reduced mus- cular effort from improved strength, reduced swelling and discomfort via improved function of the calf muscle pump and improved musculoskeletal function via increased flexibility of ankle and knee joints. ${ }^{89}$

We performed a pilot trial to obtain data on the effectiveness of exercise training to treat post-thrombotic syndrome and to assess the feasibility of performing a multicentre study to address this question.

\section{Methods}

\section{Setting and participants}

Potential study participants were identified at two Canadian study sites via physician referral and posting of patient-directed recruitment fly-
Competing interests: Please see end of article.

This article has been peer reviewed.

Correspondence to: Dr. Susan R. Kahn, susan.kahn@mcgill.ca

CMAJ 2011. DOI:10.1503 /cmaj.100248 
ers. Potentially eligible patients were those aged 18-75 years with unilateral, symptomatic deep venous thrombosis objectively diagnosed at least six months previously and current ipsilateral manifestations in the leg consistent with postthrombotic syndrome. Patients meeting these criteria were invited to a screening visit, where a trained research coordinator administered the Villalta scale for assessing post-thrombotic syndrome. ${ }^{10}$ Those with a score of five or greater were classified as having post-thrombotic syndrome. Patients were ineligible if they had contraindications to exercise training (e.g., lowerextremity arthritis, angina, severe obstructive lung disease), had a life expectancy of less than six months, were pregnant or lactating, had an open venous leg ulcer, were not English- or French-speaking, were geographically inaccessible for follow-up visits, or were unwilling or unable to provide signed informed consent.

Before randomization, potentially eligible patients were given a physician-supervised exercise stress test using the modified Bruce ramp protocol. ${ }^{11}$ Patients whose exercise test was stopped for reasons other than fatigue alone (e.g., severe dyspnea, chest pain, electrocardiographic coronary ischemia or arrhythmia) were excluded from the trial. ${ }^{12}$ Written informed consent was obtained from all patients before participation in the study, and ethics approval was obtained from the Jewish General Hospital Research Ethics Board and the Ottawa Hospital Research Ethics Board.

\section{Study protocol}

\section{Randomization and blinding}

Patients were randomized to exercise training or control via a web-based program (Dacima Software Inc.) that ensured concealment of treatment allocation. Randomization was stratified by study centre. Each study site had two research assistants, one unblinded and one blinded to treatment allocation. The unblinded assistant notified patients of their allocated treatment only after the baseline assessment was completed, interacted with the exercise training facility to arrange appointments for exercise-training patients and administered the control treatment to control patients. The blinded assistant performed the baseline, three-month and six-month assessments of all patients, who were instructed not to reveal their allocated treatment.

\section{Intervention and control treatments}

The intervention was an exercise training program consisting of a six-month program with strengthening, stretching and aerobic components designed to improve leg strength, leg flexibility and overall cardiovascular fitness. Patients were asked to attend 15 one-on-one sessions with an exercise trainer ( 3 sessions per week in the first two weeks, 2 per week in the third week, 1 per week in the fourth week and 1 per month thereafter). The first three sessions lasted 60 minutes, and subsequent sessions lasted 45 minutes.

During the first session, patients were given an individualized exercise prescription and a supervised training session. Exercise prescriptions followed the guidelines of the American College of Sports Medicine for achieving and maintaining cardio-respiratory fitness (60-120 minutes per week of aerobic exercise performed to within $60 \%-85 \%$ of maximal heart rate). ${ }^{13}$ Prescriptions were individualized based on results of the pre-randomization exercise stress test and the type of aerobic activity preferred (e.g., walking, jogging). Patients were instructed to do the strengthening program three to four times per week, the stretching program seven times per week and the aerobics program for 60 120 minutes per week. Each patient was given a digital heart-rate monitor to verify that he or she was training at the prescribed intensity level, and was asked to keep a daily log of frequency, duration and type of exercise performed.

In the second and subsequent sessions, the trainer provided support, addressed difficulties and gradually increased exercise intensity. After a few sessions, if the trainer judged that the patient understood the principles of modifying the program to ensure a training effect continued to be achieved, some face-to-face sessions were replaced by phone calls.

The control treatment was a standardized, one-hour educational slide presentation on postthrombotic syndrome followed by phone calls at one, two, four and five months to inquire about general well-being and leg-related symptoms. It aimed to simulate the attention and contact received by exercise training patients. Control group patients were asked not to alter their usual level of physical activity during the study.

Wearing elastic compression stockings during exercise has neither harmful nor beneficial effects in patients with post-thrombotic syndrome. ${ }^{14}$ We did not require patients to wear compression stockings during the trial; however, we documented their use.

\section{Outcomes}

\section{Feasibility indicators}

Criteria were established a priori as indicators of the feasibility of our study design (Appendix 1, available at www.cmaj.ca/cgi/content/full /cmaj.100248/DC1). 


\section{Effectiveness of intervention}

The following outcomes were assessed in all patients at baseline and at the three- and sixmonth visits by the blinded study coordinator, who received standardized training on performing these measures:

Quality of life: Venous disease-specific quality of life (the primary outcome) was measured using the validated Venous Insufficiency Epidemiological and Economic Study Quality of Life (VEINES-QOL) questionnaire. ${ }^{15} \mathrm{~A}$ difference of three points is considered clinically relevant. ${ }^{15,16}$ Generic quality of life was measured using the Short-Form Health Survey-36 (SF-36) questionnaire, ${ }^{17}$ which produces two (physical component and mental component) summary scores that reflect physical status and mental health status. A difference of four points is considered clinically relevant. ${ }^{17}$ For both measures, lower scores indicate poorer quality of life.

Severity of post-thrombotic syndrome: The Villalta scale, a reliable and valid standard to measure post-thrombotic syndrome, ${ }^{1018}$ was used to grade the severity of post-thrombotic syndrome. This scale rates the intensity, from 0 to 3 , of five venous symptoms and six signs. Points are summed into a total score. The Villalta scale yields a continuous score (the co-primary outcome; range $0-33$ ) that can also be used to categorize the severity of postthrombotic syndrome (none $=$ a score of $0-4$; mild $=5-9$; moderate $=10-14$; severe $=\geq 15$ or presence of ulcer). ${ }^{10}$

Leg strength: Strength of the gastrocnemiussoleus calf muscle complex was measured with a Haberometer device (Michael Haber-Scientific Animation, Illustration and Webdesign, Montréal, Quebec), using a simple and reliable heellift test. ${ }^{19}$ When the patient can no longer achieve the required heel-lift height or rate of lifting, the test is stopped and the total number of heel lifts performed is recorded.

Leg flexibility: Flexibility was assessed in the quadriceps, hamstring, gastrocnemius and soleus muscles with a handheld calibrated portable inclinometer (Saunders Group, Inc., Chaska, USA) using standard positions and manoeuvers. ${ }^{20}$ According to the manufacturer, the instrument is accurate to $\pm 5^{\circ}$, or $10 \%$. For each muscle group, a mean value (degrees) was recorded based on the average of three readings. For all measures, a larger angle indicates greater flexibility.

Time on treadmill: Exercise stress tests were performed before randomization and after completion of the six-month study intervention. A modified Bruce ramp protocol, designed for patients with low fitness levels, was used. ${ }^{11,21}$ Time on treadmill (in minutes) was used as an estimate of exercise capacity.

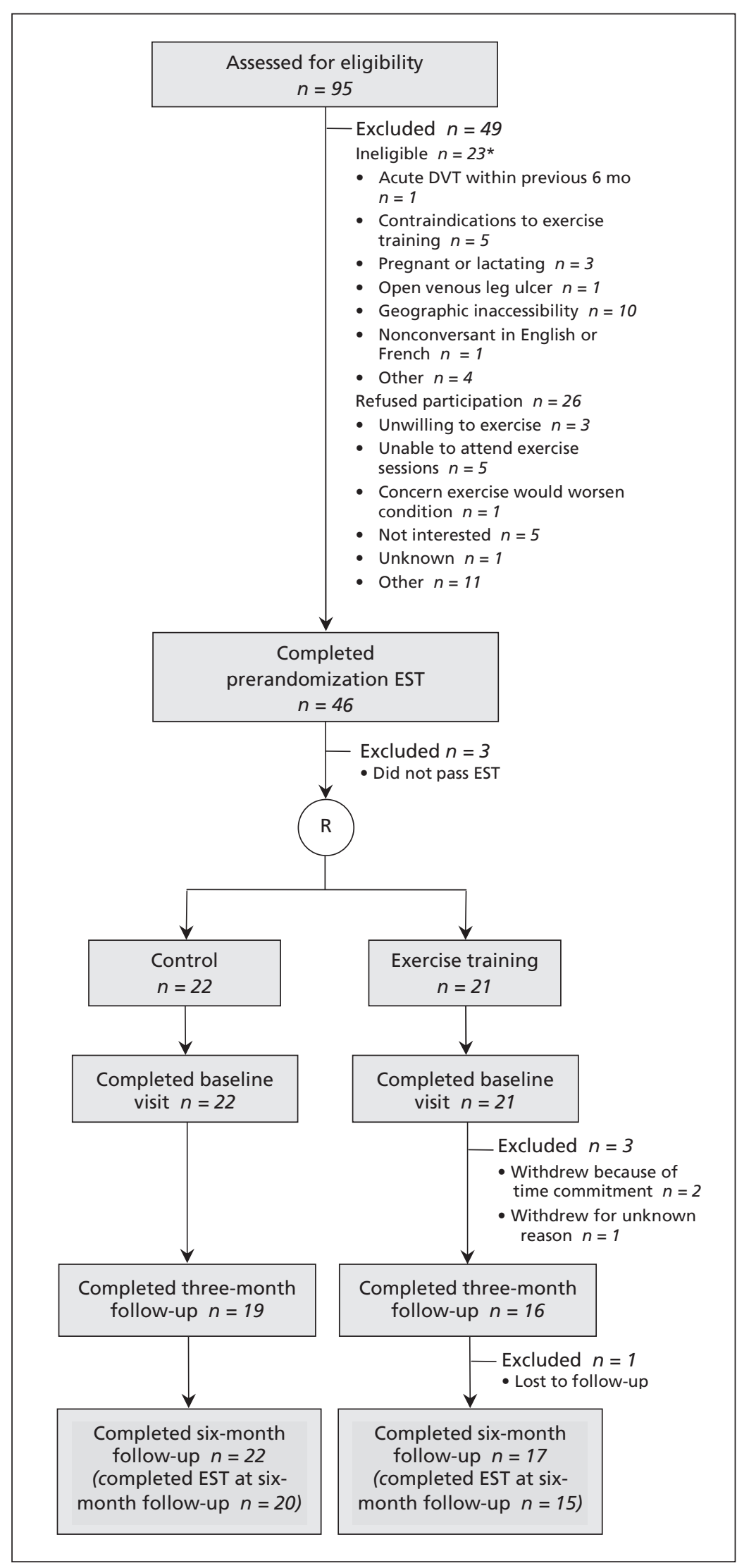

Figure 1: Consolidated Standards of Reporting Trials (CONSORT) diagram of patient flow through the trial. DVT = deep venous thrombosis, EST = exercise stress test. *More than one reason could be marked off on the screening log. 


\section{Statistical analysis}

Using a modified intent-to-treat analysis that included all participants with data at baseline and six months, we compared within-patient change from baseline to six months (mean, standard deviation [SD]) in the exercise training versus control groups in the following measures, using $t$ tests: VEINES-QOL score, Villalta score, SF-36 physical and mental component scores, number of heel lifts, stretch angles for quadriceps, hamstring, gastrocnemius and soleus muscles, and time on treadmill. Subsequently, we used analysis of covariance to perform age- and sex-adjusted comparisons of the above measures, and we conducted linear regression analysis of the effect of treatment group (exercise training $\mathrm{v}$. control) on change in VEINES-QOL score and in Villalta score, adjusted for baseline scores. Finally, we used $\chi^{2}$ tests to compare change in category of severity of post-thrombotic syndrome over time in exercise training versus control groups.

In planned exploratory analyses, we performed repeated-measures analysis of variance (ANOVA) that included data from the threemonth visit in addition to baseline and six-month data, and assessed the relation between change in VEINES-QOL score or Villalta score and change in leg strength, leg flexibility and time on treadmill.

\section{Sample size}

We aimed to generate effect sizes to aid with sample-size calculation for a larger, more definitive trial for which the likely primary outcome would be VEINES-QOL score. Based on practi-

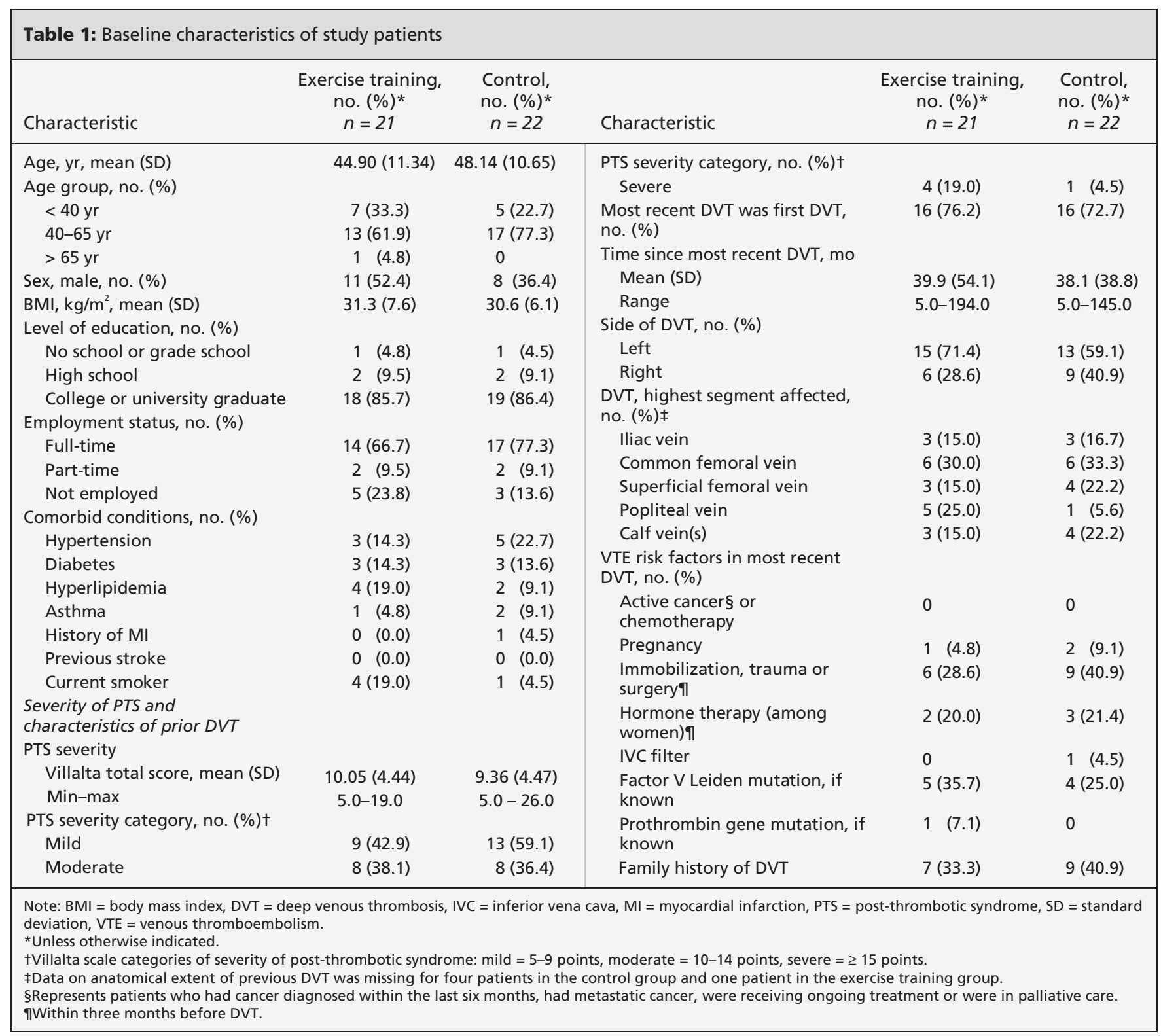


cality and cost, we aimed to enroll 44 patients, which was expected to provide a $95 \%$ confidence interval (CI) half-width of about \pm 2.4 points for change in VEINES-QOL scores, assuming an SD of this difference of four points. ${ }^{16}$

\section{Results}

\section{Patient flow and baseline characteristics}

From 2007 to 2008, 95 patients were screened, of whom 69 (73\%) met the eligibility criteria for inclusion in the study (Figure 1). Of these, 43 (62\%) consented to participate (21 randomized to exercise training, 22 to control). Three exercise training patients withdrew between baseline and three months (two because of time constraints and one for unknown reasons), and one was lost to follow-up after the three-month visit. There were no adverse events in either group.

Baseline characteristics of the study participants are shown in Table 1. Mean age was 47 years. Post-thrombotic syndrome was mild in $51.2 \%$, moderate in $37.2 \%$ and severe in $11.6 \%$ of patients. The two groups were well balanced on post-thrombotic syndrome severity, but the exercise training group had a higher proportion of men and higher levels of self-reported habitual physical activity at baseline (shown in table of Appendix 1).

\section{Adherence to allocated treatment}

Detailed results related to adherence to allocated treatment by participants are reported in Appendix 1.

\section{Effectiveness of exercise training intervention}

The effectiveness of exercise training, expressed for each outcome as the difference in within-patient change from baseline to six months in exercise training patients versus control patients, is shown in Table 2. For the primary outcome, VEINES-QOL score, the mean difference in score from baseline to six months was 6.0 (SD 5.1) among exercise training patients versus 1.4 (SD 7.2) among control group patients, with a between-group difference of 4.6 points ( $95 \%$ CI 0.54 to $8.7, p=0.027$ ) in favour of exercise training.

For severity of post-thrombotic syndrome (the co-primary outcome), the mean difference in score from baseline to six months was -3.6 (SD 3.7) among exercise training patients versus -1.6 (SD 4.3) among control patients, with a betweengroup difference of -2.0 points $(95 \% \mathrm{CI}-4.6$ to $0.6, p=0.14$ ) in favour of exercise training. Results in favour of exercise training were also obtained for between-group differences in the

\begin{tabular}{|c|c|c|c|c|c|c|c|c|c|c|c|c|c|c|}
\hline 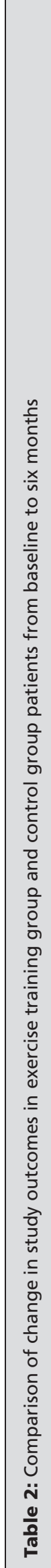 & 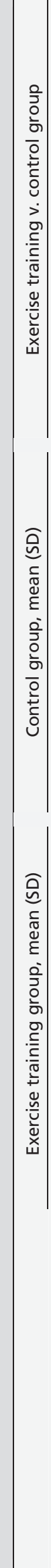 & 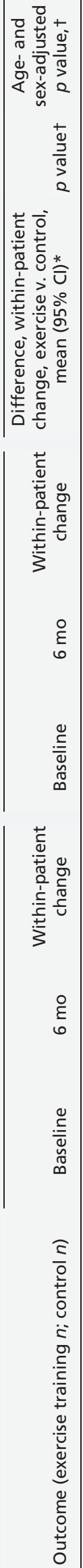 & . & 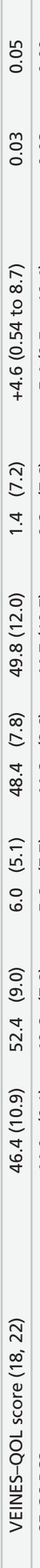 & 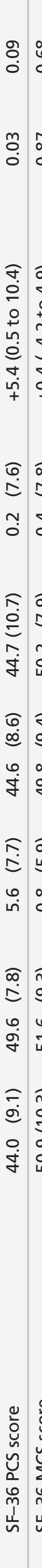 & 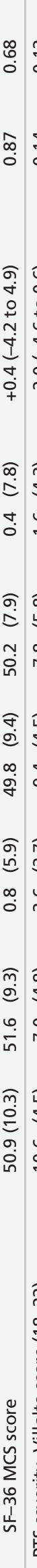 & 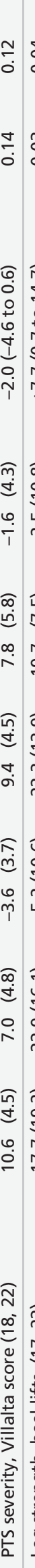 & 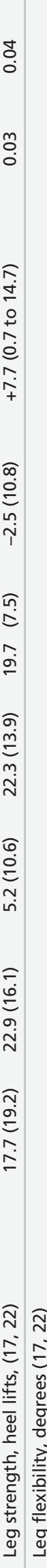 & 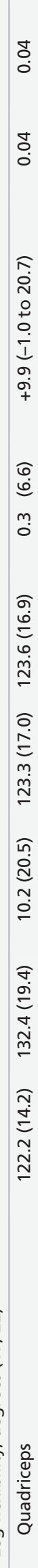 & 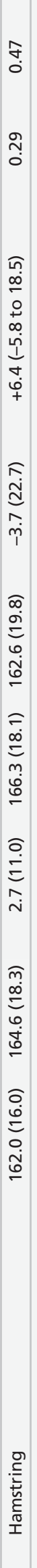 & 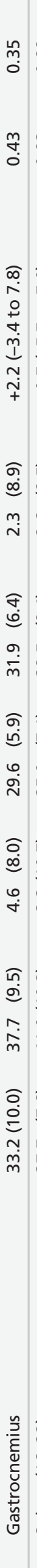 & 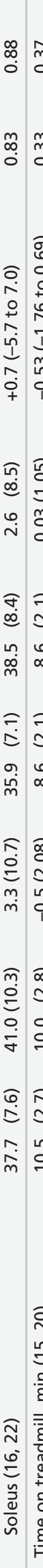 & 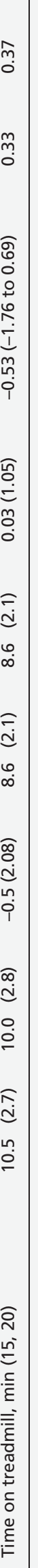 & 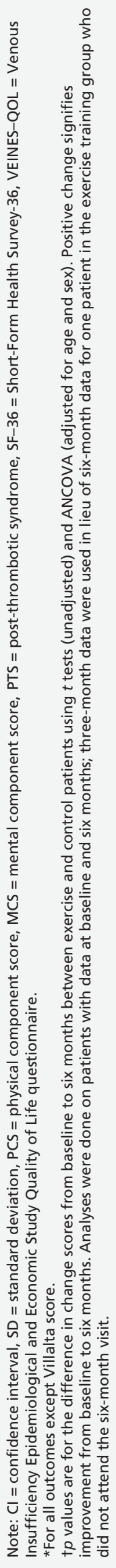 \\
\hline
\end{tabular}


physical component score on the SF-36 (5.4 points, $95 \%$ CI 0.5 to $10.4, p=0.03$ ), leg strength (7.7 heel lifts, 95\% CI 0.7 to $14.7, p=0.03$ ) and quadriceps flexibility (9.9 degrees, $95 \%$ CI -1.0 to $20.7, p=0.04$ ). Adjustment for age and sex gave similar results (Table 2). Hamstring and gastrocnemius flexibility also tended to improve more in the exercise training group, though not significantly. There was no difference between groups in change in time on treadmill.

Results were qualitatively similar when repeated-measures ANOVA incorporating data from the three-month visit was performed (data not shown) and when linear regression was performed (VEINES-QOL 4.23 points, 95\% CI 0.06 to $8.64, p=0.034$; Villalta scale -1.82 points, $95 \%$ CI -4.56 to $0.94, p=0.18$ ). Finally, exercise training patients were more likely to show improvement in category of severity of post-thrombotic syndrome, with greater improvement seen at three months (exercise training 65\% improved v. control 26\%, $p=$ 0.021 ) than at six months (exercise training $61 \%$ improved v. control $45 \%, p=0.32$ ).

With regard to potential mechanisms of the training effect, plots of change in VEINES-QOL score or Villalta score from baseline to sixmonth visit against change in number of heel lifts, flexibility of the various muscle groups or time on treadmill did not show any significant correlations ( $R^{2}$ ranged from 0.003 to 0.086 ).

Use of elastic compression stockings was reported by $57.1 \%$ of patients in the exercise training group at baseline, by $42.9 \%$ at three months and by $52.4 \%$ at six months. Stocking use was reported by $72.7 \%$ of control group patients at baseline, $50 \%$ at three months and $68.2 \%$ at six months.

\section{Interpretation}

Results of our trial suggest that a six-month exercise training program designed to increase leg strength, leg flexibility and overall fitness may be an effective treatment for post-thrombotic syndrome, with improvement in many measures, particularly venous disease-specific quality of life and severity of post-thrombotic symptoms and signs.

Although the rate of patient dropout was low, all dropouts occurred in the exercise training group. An exercise program that requires less time with an exercise trainer and can be done independently at home or work may lead to better adherence. The design of our training program was drawn from studies of patients with arterial claudication, where maximal improvement occurred with sessions at least three times weekly for more than 30 minutes and continued for six months or longer ${ }^{6.7}$ In future studies involving patients with post-thrombotic syndrome, it would be worth investigating potential benefits of programs of shorter duration (e.g., three months) and evaluation of carryover or, conversely, washout effects once training is completed.

In exploring potential mechanisms for the effectiveness of exercise, we did not find associations between improvement in leg strength, leg flexibility or time on treadmill and improvement in VEINES-QOL score or severity of post-thrombotic syndrome. Mechanisms of improvement in the exercise group may not be attributable to increased exercise capacity, or the exercise group may have had improved individual anaerobic threshold that was not captured as a change in treadmill time. Perhaps the combined effect of increased leg strength and leg flexibility explains, at least in part, the improvement noted in the exercise training group. It is also possible that a physiologic effect was mediated through psychobiological (i.e., placebo) mechanisms. ${ }^{22}$

Our patients had post-thrombotic syndrome of varying severity and were likely representative of outpatients with this condition. We excluded patients older than 75 years because we were uncertain how well the exercise-based intervention would be tolerated. The average age of our participants was similar to that in other trials of interventions to treat post-thrombotic syndrome, ${ }^{23,24}$ but it was about eight years younger than that of recent cohorts of unselected patients with deep venous thrombosis, ${ }^{25,26}$ which suggests that younger patients with post-thrombotic syndrome may be more motivated than older patients to participate in treatment studies.

Two previous small trials assessed structured exercise programs in patients with venous disease. In the first, 72 patients with acute venous thrombosis were randomly assigned to daily walking plus physiotherapist-supervised exercise sessions, or to no exercise. At six months, vein recanalization, leg circumference and quality of life improved similarly in both groups. ${ }^{27}$ Exercise was of lower frequency and duration than in our study, and adherence was not reported. In the second trial, 30 men with severe chronic venous insufficiency of diverse causes were randomly assigned to six months of exercise training (three months with supervision, then three months without supervision) or to no exercise. Exercise training improved calf muscle strength and pump function, but not valvular reflux, venous severity scores or quality of life. ${ }^{28}$ Adherence to exercise in the second trial was similar to that in our study, but patients were older, only half had post-thrombotic syndrome and women were excluded; hence, it is difficult to compare its results with ours. 


\section{Limitations}

The findings of our small trial should be interpreted with caution and require confirmation in a larger study. ${ }^{29,30}$ Given that most of our patients were young, well-educated and active (Appendix 1), the results we obtained may not be generalizable to patients with post-thrombotic syndrome at other centres.

\section{Conclusion}

In this two-centre, randomized controlled trial of a six-month exercise training program involving patients with post-thrombotic syndrome, our trial design was shown to be feasible and our exercise training intervention achieved an effect that was clinically and statistically (for some outcomes) significant. These results provide the rationale to move forward with a larger, definitive trial of exercise training to treat post-thrombotic syndrome.

\section{References}

1. Kahn SR, Ginsberg JS. Relationship between deep venous thrombosis and the postthrombotic syndrome. Arch Intern Med 2004; 164:17-26.

2. Kahn SR, Hirsch A, Shrier I. Effect of post-thrombotic syndrome on health-related quality of life after deep venous thrombosis. Arch Intern Med 2002;162:1144-8.

3. Kahn SR, Ducruet T, Lamping DL, et al. Prospective evaluation of health-related quality of life in patients with deep venous thrombosis. Arch Intern Med 2005; 165:1173-8.

4. Kahn SR. How I treat postthrombotic syndrome. Blood 2009;114:4624-31.

5. Gardner AW, Poehlman ET. Exercise rehabilitation programs for the treatment of claudication pain. A meta-analysis. JAMA 1995;274:975-80.

6. McDermott MM, Ades P, Guralnik JM, et al. Treadmill exercise and resistance training in patients with peripheral arterial disease with and without intermittent claudication: a randomized controlled trial. JAMA 2009;301:165-74.

7. Kahn SR, Shrier I, Kearon C. Physical activity in patients with deep venous thrombosis: A systematic review. Thromb Res 2008;122:763-73.

8. Araki CT, Back TL, Padberg FT, et al. The significance of calf muscle pump function in venous ulceration. J Vasc Surg 1994; 20:872-9.

9. Yang D, Vandongen YK, Stacey MC. Effect of exercise on calf muscle pump function in patients with chronic venous disease. Br J Surg 1999;86:338-41.

10. Kahn SR, Partsch H, Vedantham S, et al. Definition of postthrombotic syndrome of the leg for use in clinical investigations: a recommendation for standardization. J Thromb Haemost 2009; 7:879-83.

11. Kaminsky LA, Whaley MH. Evaluation of a new standardized ramp protocol: the BSU/Bruce Ramp protocol. J Cardiopulm Rehabil 1998; 18:438-44.

12. Darrow MD. Ordering and understanding the exercise stress test. Am Fam Physician 1999;59:401-10.

13. American College of Sports Medicine Position Stand. The recommended quantity and quality of exercise for developing and maintaining cardiorespiratory and muscular fitness, and flexibility in healthy adults. Med Sci Sports Exerc 1998;30:975-91.

14. Kahn SR, Azoulay L, Hirsch A, et al. Effect of graduated elastic compression stockings on leg symptoms and signs during exercise in patients with deep venous thrombosis: a randomized cross-over trial. J Thromb Haemost 2003;1:494-9.

15. Lamping DL, Schroter S, Kurz X, et al. Evaluating outcomes in chronic venous disorders of the leg: Development of a scientifically rigorous, patient-reported measure of symptoms and quality of life. J Vasc Surg 2003;37:410-9.

16. Kahn SR, Lamping DL, Ducruet T, et al. VEINES-QOL/Sym questionnaire was a reliable and valid disease-specific quality of life measure for deep venous thrombosis. J Clin Epidemiol 2006;59:1049-56

17. Ware JE, Kosinski M, Keller S. SF-36 physical and mental sum- mary measures: A user's manual. Boston (MA): The Health Institute, New England Medical Center; 1994.

18. Kahn SR. Measurement properties of the Villalta scale to define and classify the severity of the post-thrombotic syndrome. $J$ Thromb Haemost 2009;7:884-8.

19. Haber M, Golan E, Azoulay L, et al. Reliability of a device measuring triceps surae muscle fatigability. Br J Sports Med 2004;38:163-7.

20. Ekstrand J, Wiktorsson M, Oberg B, et al. Lower extremity goniometric measurements: a study to determine their reliability. Arch Phys Med Rehabil 1982;63:171-5.

21. Will PM, Walter JD. Exercise testing: improving performance with a ramped Bruce protocol. Am Heart J 1999;138:1033-7.

22. Crum AJ, Langer EJ. Mind-set matters: exercise and the placebo effect. Psychol Sci 2007;18:165-71.

23. O'Donnell MJ, McRae S, Kahn SR, et al. Evaluation of a venous-return assist device to treat severe post-thrombotic syndrome (VENOPTS): A randomized controlled trial. Thromb Haemost 2008;99:623-9.

24. Ginsberg JS, Hirsh J, Julian J, et al. Prevention and treatment of postphlebitic syndrome: results of a 3-part study. Arch Intern Med 2001;161:2105-9.

25. Kahn SR, Shrier I, Julian JA, et al. Determinants and time course of the post-thrombotic syndrome after acute deep venous thrombosis. Ann Intern Med 2008; 149:698-707.

26. Tick LW, Kramer MH, Rosendaal FR, et al. Risk factors for postthrombotic syndrome in patients with a first deep venous thrombosis. J Thromb Haemost 2008;6:2075-81.

27. Isma N, Johanssson E, Bjork A, et al. Does supervised exercise after deep venous thrombosis improve recanalization of occluded vein segments? A randomized study. J Thromb Thrombolysis 2007;23:25-30.

28. Padberg FT Jr, Johnston MV, Sisto SA. Structured exercise improves calf muscle pump function in chronic venous insufficiency: a randomized trial. J Vasc Surg 2004;39:79-87.

29. Arnold DM, Burns KE, Adhikari NK, et al. The design and interpretation of pilot trials in clinical research in critical care. Crit Care Med 2009;37:S69-74.

30. Lancaster GA, Dodd S, Williamson PR. Design and analysis of pilot studies: recommendations for good practice. J Eval Clin Pract 2004;10:307-12.

Competing interests: Susan Kahn is principal investigator of an ongoing trial funded by the Canadian Institutes for Health Research (CIHR) investigating active versus placebo compression stockings to prevent post-thrombotic syndrome after deep venous thrombosis, for which Sigvaris Corp. has provided active and placebo compression stockings. She is a coapplicant and steering committee member on an NIHfunded trial (funded by the United States National Institutes of Health) of pharmacomechanical catheter-directed thrombolysis plus standard anticoagulation alone. She has received honoraria to speak on post-thrombotic syndrome at various national and international academic conferences. Clive Kearon is principal investigator for a CIHR grant for the evaluation of a D-dimer-based management strategy for selecting patients with unprovoked venous thromboembolism for indefinite anticoagulation. He is a steering committee member for one recently completed and two ongoing studies of venous thromboembolism treatment by Boehringer Ingelheim. Marc Rodger served on an advisory board for Boehringer Ingelheim, Sanofi-Aventis and bioMérieux. He is appointed at the Ottawa Hospital Research Institute, which received compensation from Boehringer Ingelheim, Sanofi-Aventis, bioMérieux and LEO Pharma for this service. No competing interests declared by Ian Shrier, Stan Shapiro, Adrielle Houweling, Andrew Hirsch, Robert Reid, Khalil Rabhi, Michael Kovacs, David Anderson and Philip Wells.

Affiliations: From the Department of Medicine (Kahn, Hirsch) and the Department of Epidemiology and Biostatistics (Shapiro), McGill University; the Centre for Clinical Epidemiology and Community Studies (Kahn, Shrier, Shapiro, Houweling) Jewish General Hospital; and the Department of Chest Medicine (Rabhi), Hôpital du SacréCoeur de Montréal, Montréal, Que.; Minto Prevention and Rehabilitation Centre (Reid), University of Ottawa Heart Institute; the Departments of Medicine and Department of 
Epidemiology and Community Medicine (Rodger, Wells), University of Ottawa, Ottawa, Ont.; the Department of Medicine (Kearon), McMaster University, Hamilton, Ont.; the Department of Medicine (Kovacs), University of Western Ontario, London, Ont.; and the Department of Medicine (Anderson), Dalhousie University, Halifax, NS

Contributors: Susan Kahn, Ian Shrier and Clive Kearon were responsible for the conception and design of the study. Stan Shapiro, Andrew Hirsch, Robert Reid, Marc Rodger, Michael Kovacs, David Anderson and Philip Wells were involved in the design of the study. Susan Kahn, Andrew Hirsch, Adrielle Houweling, Robert Reid, Marc Rodger and Philip Wells acquired the data. Susan Kahn, Adrielle Houweling, Khalil Rabhi, Ian Shrier and Stan Shapiro analyzed the data. Susan Kahn, Ian Shrier, Stan Shapiro and Adrielle Houweling interpreted the data. Susan Kahn and Adrielle Houweling drafted the manuscript. All of the authors critically revised the manuscript for important intellectual content, and all of them approved the final version submitted for publication. Susan Kahn had full access to all of the data in the study and takes responsibility for the integrity of the data and the accuracy of the data analysis.

Funding: This study was funded by the CIHR Randomized Controlled Trials Program (grant no. MTC82338). Susan Kahn is national investigator (chercheur national) of the Fonds de la recherche en santé du Québec (FRSQ). Ian Shrier was supported by an FRSQ Senior Clinician Scientist Award. Clive Kearon is a career investigator of the Heart and Stroke Foundation of Ontario and is supported by a CIHR Team Grant in Venous Thromboembolism (grant no. FRN 79846). Marc Rodger is a career scientist of the Heart and Stroke Foundation of Ontario and is supported by the Early Researcher Award of the Ministry of Research and Innovation. Philip Wells and Marc Rodger hold Department of Medicine Research Awards, and Philip Wells holds a Canada Research Chair in Thromboembolic Diseases.

Acknowledgements: The authors thank the patients who participated in the EXPO Trial and their research assistants, Cassy Robinson-Cohen, Alla Muladzanov, Jennifer Fleming, Anne Marie-Clement and Melissa Spero, for their involvement in patient recruitment and follow-up for the study. The authors also express their gratitude to Marla Gold, director, Sabrina Pillay, program coordinator, and the other staff members at the Cardiovascular Health Improvement Program in Montréal, as well as Ed Gannon, charge stress technologist, Diagnostic Imaging, University of Ottawa Heart Institute, for coordinating the exercise stress tests. The authors gratefully acknowledge Ilka Lowensteyn, Department of Medicine, McGill University in Montréal and the staff of Bob Reid at the Minto Prevention and Rehabilitation Centre, University of Ottawa Heart Institute in Ottawa, for their roles in implementing the exercise program for the exercise training intervention. Finally, the authors wish to acknowledge Louise Beliveau, Department of Kinesiology, Université de Montréal, and James Douketis, Department of Medicine, McMaster University, who served as members of the Data Safety Committee.

Editor's note: Portions of this work were presented at the Society for Clinical Trials 30th Annual Meeting, May 2009, Atlanta, USA; the XXII Congress of the International Society on Thrombosis and Haemostasis, July 2009, Boston, USA; and the 50th Annual Meeting of the American Society of Hematology, December 2009, New Orleans, USA.

Disclaimer: Stan Shapiro is a biostatistical consultant for $C M A J$ and was not involved in the editorial decision-making process for this article.

Editor's note: For the study protocol pertaining to this article, please see Appendix 2 (available at www.cmaj.ca/cgi/content/full/cmaj.100248/DC1).
ATIVAN is useful for the short-term relief of manifestations of excessive anxiety in patients with anxiety neurosis. It is also useful as an adjunct for the relief of excessive anxiety that might be present prior to surgical interventions. Anxiety and tension associated with the stresses of everyday life usually do not require treatment with anxiolytic drugs.

ATIVAN is contraindicated in patients with myasthenia gravis or acute narrow angle glaucoma, and in those with known hypersensitivity to benzodiazepines.

Severe anaphylactic/anaphylactoid reactions have been reported with the use of benzodiazepines. Cases of angioedema involving the tongue, glottis or larynx have been reported in patients after taking the first or subsequent doses of benzodiazepines. Some patients taking benzodiazepines have had additional symptoms such as dyspnea, throat closing or nausea and vomiting. Some patients have required medical therapy in the emergency department. If angioedema involves the tongue, glottis or larynx, airway obstruction may occur and be fatal. Patients who develop angioedema after treatment with a benzodiazepine should not be rechallenged with the drug.

ATIVAN is not recommended for use in depressive neurosis or in psychotic reactions. Because of the lack of sufficient clinical experience, lorazepam is not recommended for use in patients less than 18 years of age. Since ATIVAN has a central nervous system depressant effect, patients should be advised against the simultaneous use of other CNS depressant drugs. Patients should also be cautioned not to take alcohol during the administration of lorazepam because of the potentiation of effects that may occur. ATIVAN should not be used during pregnancy. Since lorazepam is also a benzodiazepine derivative, its administration is rarely justified in women of childbearing potential. ATIVAN should not be administered to breast-feeding women, unless the expected benefit to the mother outweighs the potential risk to the infant.

Use of benzodiazepines, including lorazepam, may lead to potentially fatal respiratory depression.

Excessive sedation has been observed with lorazepam at standard therapeutic doses.

The most frequently reported adverse reaction to ATIVAN was drowsiness. See prescribing information for complete adverse reaction information.

The lowest effective dose of ATIVAN should be prescribed for the shortest duration possible. The risk of withdrawal and rebound phenomena is greater after abrupt discontinuation; therefore, the drug should be discontinued gradually. Withdrawal symptoms (e.g., rebound insomnia) can appear following cessation of recommended doses after as little as one week of therapy. Abrupt discontinuation of lorazepam should be avoided and a gradual, dose-tapering schedule followed after extended therapy.

ATIVAN should not be administered to individuals prone to drug abuse. Lorazepam may have abuse potential, especially in patients with a history of drug and/or alcohol abuse.

(92010 Pfizer Canada Inc.
Kirkland Quebec 